\title{
NK cells remember
}

Traditionally, natural killer (NK) cells have been considered to be part of the innate immune system. However, recent work has indicated that NK cells may have more traits in common with cells of the adaptive immune system than originally thought, including the concept of immunological memory. Indeed, Lanier and colleagues, in a study published in Nature, now show that NK cells go through the four phases of the adaptive immune response - proliferation, contraction, memory and recall - and draw parallels between NK-cell and T-cell responses.

The authors made use of the well-established model of murine cytomegalovirus (MCMV) infection, during which NK cells are known to confer protection by expressing the receptor Ly49H (also known as KLRA8; which recognizes the

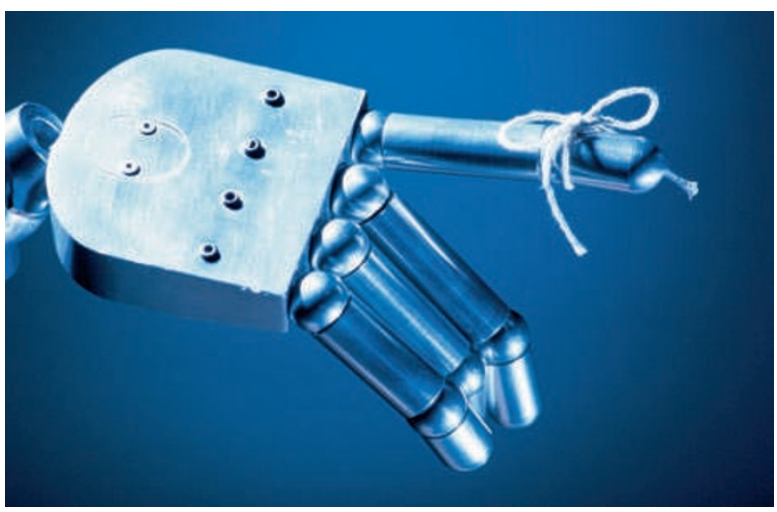

m157 MCMV protein). To examine the proliferation phase of the NK-cell response, they transferred $\mathrm{Ly}_{4} 9 \mathrm{H}^{+}$

NK cells into DAP12-deficient mice (which do not express a functional Ly49H) and observed that, following MCMV infection, only the transferred $\mathrm{Ly}_{4} 4 \mathrm{H}^{+} \mathrm{NK}$ cells were proliferating; this suggests that the response was specific. In addition, after transferring different numbers of Ly $49 \mathrm{H}^{+} \mathrm{NK}$ cells into the DAP12deficient mice, they observed comparable kinetics of expansion in the spleen and liver, indicating that precursor frequency does not affect the final NK-cell population size. Interestingly, the authors also observed a contraction phase following the expansion of the NK-cell population, which was similar to the prolonged decline in $\mathrm{CD} 4^{+} \mathrm{T}$-cell numbers after proliferation.

Following the contraction phase, the T-cell response moves into the memory phase, during which a stable memory T-cell population resides in lymphoid and non-lymphoid tissues. A population of NK cells was detectable 70 days after MCMV infection (designated 'memory' NK cells), so the authors investigated whether this population was functional. They showed that the memory NK cells produced high levels of interferon- $\gamma$ (IFN $\gamma$ ) ex vivo following reactivation. Furthermore, when compared with a naive $\mathrm{Ly} 49 \mathrm{H}^{+} \mathrm{NK}$-cell population, the memory NK cells produced higher levels of IFN $\gamma$, displayed enhanced degranulation and had a more mature phenotype (as determined by the expression of activation molecules).

Finally, the authors examined whether memory NK cells could also undergo a secondary expansion phase (or recall response), such as that which occurs during an antigenspecific T-cell response. Indeed, an expanded memory $\mathrm{Ly}_{49 \mathrm{H}^{+}} \mathrm{NK}$-cell population was readily detectable following adoptive transfer into DAP12-deficient mice that were subsequently infected with MCMV. Importantly, memory Ly $49 \mathrm{H}^{+} \mathrm{NK}$ cells conferred higher levels of protection from MCMV infection when transferred into newborn mice (which are susceptible to the infection owing to a lack of mature $\mathrm{NK}$ cells) than naive $\mathrm{Ly}_{49 \mathrm{H}^{+}} \mathrm{NK}$ cells. This indicates that, similarly to antigen-specific T cells, memory NK cells can confer high levels of protection from a subsequent infection with the same pathogen.

This study highlights that NK cells have traits of both innate and adaptive immune cells, questioning the concept of NK cells being solely innate immune cells.

Rachel David

ORIGINAL RESEARCH PAPER Sun, J. C., Beilke, J. N. \& Lanier, L. L. Adaptive immune features of natural killer cells. Nature 11 Jan 2009 (doi:10.1038/nature07665) 